\title{
Reliability Measure of a Relay Parallel System under Dependence Conditions
}

\author{
Vanderlei da Costa Bueno \\ Institute of Mathematics and Statistics, São Paulo University, São Paulo, Brasil \\ Email: bueno@ime.usp.br
}

Received September 29, 2012; revised October 30, 2012; accepted November 15, 2013

\begin{abstract}
In a relay system of dependent components, the failure to close reliability measure is given as a Girsanov transform of the failure to open reliability measure.
\end{abstract}

Keywords: Martingale Methods in Reliability Theory; Compensator Process; Parallel Operation

\section{Introduction}

As in Barlow and Proschan [1], a complex coherent reliability system is completely characterized by its structure function $\Phi=\Phi(X(t))$ assuming values in the set $\{0,1\}$ and where $X(t)=\left(X_{1}(t), X_{2}(t), \cdots, X_{n}(t)\right)$ is a stochastic process assuming values in $\{0,1\}$. The stochastic process $\left(X_{i}(t)\right)_{t \geq 0}$, represents the state of the $i$-th component. $X_{i}(t)=1$, if the component $i$ is working at time $t$ and $X_{i}(t)=0, i$ if the component $i$ is in a failed state at time $t$. Also, the system state $\Phi(X(t))$ has such an interpretation, is increasing in each coordinate and each component is relevant, that is, there is a time $t$ and a configuration of $\left(X_{i}(t)\right)_{t \geq 0}$ in which the functioning of the component $i$ is fundamental for the functioning of the system.

A relay system is subject to two kinds of failure: failure to close and failure to open. Similarly, circuits construct from these relays are subject to the same kinds of failure. If the $i$-th relay responds correctly to a command to close, $X_{i}(t)=1$, (that is, closes), and $X_{i}(t)=0$, otherwise, $i=1 ; 2$ and $\Phi(X(t))=1$ if the circuit responds correctly to a command to close (that is, closes) if, and only if, at least one of its components responds correctly to a command to close, and 0 otherwise, then,

$$
\Phi(X(t))=\max \left\{X_{1}(t), X_{2}(t)\right\}
$$

is a parallel system. Next, let $Y_{i}(t)=1$ if the $i$-th relay responds correctly to a command to open (that is open), and $Y_{i}(t)=0$ otherwise, $i=1 ; 2$. Let $\Psi(Y(t))=1$ if the circuit responds correctly to a command to open (that is, open), and 0 otherwise. Then

$$
\Psi(Y(t))=\min \left\{Y_{1}(t), Y_{2}(t)\right\}=\Phi^{D}(Y(t))
$$

a series system, the dual of $\Phi$, is the system represent- ing the correct system response to a command to open.

Generally, given a structure function $\Phi$, its dual $\Phi^{D}$ is defined by

$$
\Phi^{D}(X(t))=1-\Phi(1-X(t))
$$

where

$$
1-X(t)=\left(1-X_{1}(t), \cdots, 1-X_{n}(t)\right) .
$$

The concept of dual structure is useful in analyzing system of components subject to two kinds of failures, such as relays system and safety monitoring systems. It is interesting and useful to note that both failure to close and failure to open can be analyzed using the same structure function $\Phi$. In this paper, under dependence conditions, we analyze the dual structure probability measure of a parallel system, through a transform of the failures counting processes compensators in the original probability space. In Section 2 we analyse the problem for a parallel system of two components. In Section 3 we generalize the results and in Section 4 we discuss some reliability preservation properties.

Without loss of generality, firstly, we consider a parallel system of two components. We observe two component lifetimes $T$ and $S$, which are positive random variables defined in a complete probability $\operatorname{space}(\Omega, \mathfrak{I}, P)$ through the family of sub $\sigma$-algebras $\left(\mathfrak{I}_{t}\right)_{t \geq 0}$ of $\mathfrak{I}$, where

$$
\mathfrak{I}_{t}=\sigma\left\{1_{\{S>s\}}, 1_{\{T>s\}}, 0 \leq s \leq t\right\},
$$

$\mathfrak{I}_{0}=\{\Omega, \phi\}$ satisfies Dellacherie's conditions.

In order to simplify the notation, in this paper we assume that relations such as $\subset,=, \leq,<, \neq$ between random variables and measurable sets, always hold with probability one. In what follows we assume that $S$ and $T$ 
are totally inaccessible $\mathfrak{I}_{t}$-stopping time and that $P(S=T)=0$, that is, the lifetimes can be dependent but simultaneous failures are ruled out.

The parallel operation of $S$ and $T$ is defined by the maximum between $S$ and $T$ and denoted by

$$
S \vee T=\max \{S, T\} .
$$

If we denote the survival functions of $S$ and $T$ as $\bar{G}(t)=P\left(S>t \mid \mathfrak{I}_{t}\right)$ and $\bar{F}(t)=P\left(T>t \mid \mathfrak{I}_{t}\right)$ respectively, it follows from Arjas and Yashin [2], that, under some conditions, the $\mathfrak{I}_{t}$-compensator processes of $N_{B}(t)=1_{\{S \leq t\}}$ and $N_{A}(t)=1_{\{T \leq t\}}$ are given by

$$
B(t)=-\ln P\left(S>t \mid \mathfrak{I}_{t}\right)=-\ln \bar{G}(t \wedge S)
$$

and

$$
A(t)=-\ln P\left(T>t \mid \mathfrak{I}_{t}\right)=-\ln \bar{F}(t \wedge T) .
$$

We assume such conditions and as $S$ and $T$ are totally inaccessible-stopping time the compensator processes are continuous.

Now we calculate

$$
\begin{aligned}
& P\left(S \vee T \mid \mathfrak{I}_{t}\right) \\
& =P\left(S>t \mid \mathfrak{I}_{t}\right)+P\left(T>t \mid \mathfrak{I}_{t}\right)-P\left(S \vee T>t \mid \mathfrak{I}_{t}\right) \\
& =\mathrm{e}^{-[A(t)+B(t)]}\left\{\mathrm{e}^{A(t)}+\mathrm{e}^{B(t)}-1\right\}
\end{aligned}
$$

and therefore, in the set $\{t<S \vee T\}$ the $\mathfrak{I}_{t}$-compensator of $1_{\{S \vee T \leq t\}}$ is

$$
-\ln P\left(S \vee T>t \mid \mathfrak{I}_{t}\right)=A(t)+B(t)-\ln \left[\mathrm{e}^{A(t)}+\mathrm{e}^{B(t)}-1\right] .
$$

We intend to define compensator transformation of $A(t)$ and $B(t)$ to $A^{*}(t)$ and $B^{*}(t)$ in the way that the above expression is the sum $A^{*}(t)+B^{*}(t)$ which characterizes uniquely the lifetime of a series system and, therefore, the dual of such a parallel system. As this operation is symmetric on $S$ and $T$, the idea is to combine compensator transformations in $A(t)$ and $B(t)$.

Firstly, we consider the compensator transform

$$
\begin{aligned}
& A^{*}(t)=\int_{0}^{t} \frac{\mathrm{e}^{B(s)}-1}{\mathrm{e}^{A(s)}+\mathrm{e}^{B(s)}-1} \mathrm{~d} A(s) \\
& =\int_{0}^{t}\left[1-\frac{\mathrm{e}^{A(s)}}{\mathrm{e}^{A(s)}+\mathrm{e}^{B(s)}-1}\right] \mathrm{d} A(s)=A(t)-\int_{0}^{t} \frac{\mathrm{e}^{A(s)}}{\mathrm{e}^{A(s)}+\mathrm{e}^{B(s)}-1} \mathrm{~d} A(s) .
\end{aligned}
$$

To prove the main Theorem of this section we are going to use the following Lemma:

\section{Lemma 2.1}

Under the above hypothesis the following process

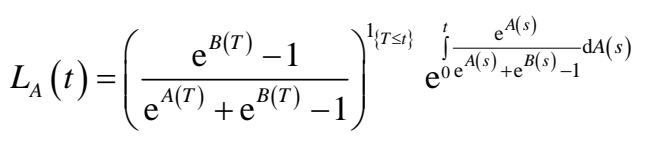

is a nonnegative local $\mathfrak{I}_{t}$-martingale and $E\left[L_{A}(t)\right]=1$.

Proof

We consider the localization sequence, the $\mathfrak{I}_{t}$-stopping time defined

By

$$
V_{n}=\inf \{t \geq 0: A(t) \geq n \text { or } B(t) \geq n\} .
$$

It is sufficient to prove that the process

$$
L_{A}^{n}(t)=\left(\frac{\mathrm{e}^{B(T)}-1}{\mathrm{e}^{A(T)}+\mathrm{e}^{B(T)}-1}\right)^{1_{\left\{T \leq t \wedge V_{n}\right\}}} \mathrm{e}^{t \wedge N_{0}} \frac{\mathrm{e}^{\mathrm{A}(s)}}{\mathrm{e}^{\mathrm{A}(s)}+\mathrm{e}^{B(s)}-1} \mathrm{dA}(s)
$$

is a bounded $\mathfrak{J}_{t}$-martingale.

For any $\mathfrak{I}_{t}$-stopping time $V \leq V_{n}$ we can write

$$
\begin{aligned}
& L_{A}^{n}(V) \\
& =1-\int_{0}^{V} \mathrm{e}^{\mathrm{s} \mathrm{e}^{\mathrm{A}(u)}+\mathrm{e}^{\mathrm{A}(u)}} \mathrm{dA} \mathrm{e}^{B(u)} \frac{\mathrm{e}^{\mathrm{A}(s)}}{\mathrm{e}^{\mathrm{A}(s)}+\mathrm{e}^{B(s)}-1} \mathrm{~d}(N(s)-A(s)) .
\end{aligned}
$$

The procedure is easy: On the set $\{V<T\}$ we have

$$
\begin{aligned}
& L_{A}^{n}(V) \\
& =1+\int_{0}^{V} \mathrm{e}^{\mathrm{s} \mathrm{e}^{\mathrm{A}\left(\mathrm{e}^{\mathrm{A}(u)}+\mathrm{e}^{\left(u^{(u)}-1\right.}\right.} \mathrm{dA}(u)} \frac{\mathrm{e}^{\mathrm{A}(s)}}{\mathrm{e}^{\mathrm{A}(s)}+\mathrm{e}^{B(s)}-1} \mathrm{~d} A(s)
\end{aligned}
$$

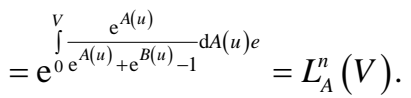

Otherwise, on the set $\{V \geq T\}$,

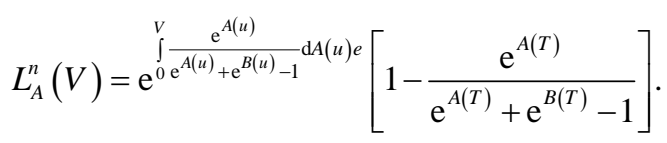

As the integrand

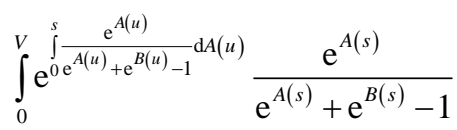

is an $\mathfrak{I}_{t}$-predictable process and $N(s)-A(s)$ is an $\mathfrak{I}_{t}$-martingale, $L_{A}^{n}(t)$ is an $\mathfrak{I}_{t}$-martingale with $E\left[L_{A}^{n}(t)\right]=1$.

Secondly, we consider the compensator transform

$B^{*}(t)=\int_{0}^{t} \frac{\mathrm{e}^{A(s)}-1}{\mathrm{e}^{A(s)}+\mathrm{e}^{B(s)}-1} \mathrm{~d} B(s)=B(t)-\int_{0}^{t} \frac{\mathrm{e}^{B(s)}}{\mathrm{e}^{A(s)}+\mathrm{e}^{B(s)}-1} \mathrm{~d} B(s)$.

and with the same argument to prove Lemma 2.1 we can prove Lemma 2.2:

Lemma 2.2 Under the above hypothesis the following process

$$
L_{B}(t)=\left(\frac{\mathrm{e}^{A(S)}-1}{\mathrm{e}^{A(T)}+\mathrm{e}^{B(T)}-1}\right)^{1_{\{S s t\}}} \mathrm{e}^{\int^{0} \frac{\mathrm{e}^{B(s)}+\mathrm{e}^{B(s)}-1}{B(s)} \mathrm{d}(s)}
$$

$L_{B}^{n}(t)$ is a nonnegative $\mathfrak{I}_{t}$-martingale with 


$$
E\left[L_{B}^{n}(t)\right]=1 .
$$

Observe that the same expression for the $\mathfrak{I}_{t}$-compensator of $1_{\{S \vee T \leq t\}}$ is obtained through the transformation:

$$
\begin{aligned}
& A^{*}(t)+B^{*}(t) \\
= & A(t)+B(t)-\int_{0}^{t} \frac{\mathrm{e}^{A(s)} \mathrm{d} A(s)+E^{B(s)} \mathrm{d} B(s)}{\mathrm{e}^{A(s)}+\mathrm{e}^{A(s)}-1} \\
= & A(t)+B(t)-\ln \left[\mathrm{e}^{A(t)}+\mathrm{e}^{B(t)}-1\right] .
\end{aligned}
$$

Then, we propose the compensator transforms:

$$
\begin{aligned}
& A^{*}(t)=\int_{0}^{t} \alpha(s) \mathrm{d} A(s), \\
& \alpha(s)=\frac{\mathrm{e}^{B(s)}-1}{\mathrm{e}^{A(s)}+\mathrm{e}^{B(s)}-1},
\end{aligned}
$$

and

$$
B^{*}(t)=\int_{0}^{t} \beta(s) \mathrm{d} B(s), \beta(s)=\frac{\mathrm{e}^{\mathrm{A}(s)}-1}{\mathrm{e}^{A(s)}+\mathrm{e}^{B(s)}-1},
$$

to prove the main Theorem:

Theorem 2.3 Under the above hypothesis the following process

$$
\begin{aligned}
L(t) & =L_{A}(t) L_{B}(t) \\
& =(\alpha(T))^{1_{\{T \leq t\}}}(\beta(S))^{1_{\{}\{S t\}}\left[\mathrm{e}^{A(t)}+\mathrm{e}^{B(t)}-1\right]
\end{aligned}
$$

is a nonnegative local $\mathfrak{J}_{t}$-martingale and $E[L(t)]=1$.

Proof. Using Lemma 2.1, Lemma 2.2 and the Stieltjes differentiation rule we have

$$
\begin{aligned}
& L_{A}(t) L_{B}(t)-1 \\
= & \int_{0}^{t} L_{A}(s-d) L_{B}(s)+\int_{0}^{t} L_{B}(s-d) L_{A}(s) \\
& +\sum_{s \leq t} \Delta L_{A}(s) \Delta L_{B}(s) .
\end{aligned}
$$

As by assumption, $A(t)$ and $B(t)$ are continuous and $P(S=T)=0$ we have

$$
\sum_{s \leq t} \Delta L_{A}(s) \Delta L_{B}(s)=0
$$

and therefore $L A(t) L B(t)$ in an local $\mathfrak{I}_{t}$-martingale with $E[L A(t) L B(t)]=1$ and the theorem is proved.

Now, we are looking for a probability measure $Q$, such that, under $Q, C^{*}(t)=A^{*}(t)+B^{*}(t)$ becomes the $\mathfrak{I}_{t}$ compensator of $1_{\{S \vee T \leq t\}}$ with respect to this modified probability measure.

Under certain conditions, it is possible to find $Q$. Indeed, assume that the process $L(t)$ is uniformly integrable. Then it follows from well known results on point process martingales (Girsanov Theorem,Bremaud [3]) that the desired measure $Q$ is given by the Radon Nikodyn derivative

$$
\frac{\mathrm{d} Q}{\mathrm{~d} P}=L(\infty)
$$

\section{Remark:}

In the case where $T$ and $S$ are identically distributed, we have $A(t)=B(t)$ and the compensator transform is given by

$$
A^{*}(t)+B^{*}(t)=2 \int_{0}^{t} \frac{\mathrm{e}^{A(s)}-1}{2 \mathrm{e}^{A(s)}-1} \mathrm{~d} A(s)=\int_{0}^{t} \frac{2-2 \mathrm{e}^{-A(s)}}{2-\mathrm{e}^{-A(s)}} \mathrm{d} A(s),
$$

which is used in Bueno and Carmo [4], to de_ne active redundancy operation when the component and the spare are dependent but identically distributed.

Parallel operations are very important in reliability theory: the performance of a parallel system are always better than the performance of any coherent system with the same components; it is used in replacement models, to optimize system reliability through active redundancy. However, if the component are stochastically dependent the reliability of a coherent system is a difficult and tedious calculation involving multivariate distributions. The calculation becomes more tractable under the assumption of a series system, in which case the reliability is the survival function of a multivariate positive random vector. It is also can be easily done through the compensator processes. We can show this argument easily:

\section{Corollary 2.4}

Under the hypothesis of Theorem 2.3, and under $Q$ such that

$$
\frac{\mathrm{d} Q}{\mathrm{~d} P}=L(\infty),
$$

we have

$$
Q(S \wedge T>t)=P(S \vee T>t), t \geq 0 .
$$

Proof As the compensators are deterministic before any failure, we can write:

$$
\begin{aligned}
Q(S \wedge T>t) & =E_{Q}\left[1_{\{S \wedge T>t\}}\right]=E_{P}\left[L(\infty) 1_{\{S \wedge T>t\}}\right] \\
& =E_{P}\left\{E_{P}\left[L(\infty) 1_{\{S \wedge T>t\}} \mid \mathfrak{J}_{t}\right]\right\} \\
& =E_{P}\left\{1_{\{S \wedge T>t\}} E_{P}\left[L(\infty) \mid \mathfrak{J}_{t}\right]\right\} \\
& =E_{P}\left[1_{\{S \wedge T>t\}} L(t)\right] \\
& =E_{P}\left[1_{\{S \wedge T>t\}}\left(\mathrm{e}^{A(t)}+\mathrm{e}^{B(t)}-1\right)\right] \\
& =\left(\mathrm{e}^{A(t)}+\mathrm{e}^{B(t)}-1\right) P(S \wedge T>t) \\
& =P(S \vee T>t) .
\end{aligned}
$$

As an application we calculate the Barlow and Proschan [1] reliability importance of a component for the system. In the independent and absolutely continuous case, it is the probability that the component causes the 
system failure. For dependent components, this quantity is

$$
\begin{aligned}
& I_{P}(S)=P(S=S \vee T)=P(S \geq S \vee T) \\
& =P(S \geq T)=1-P(S \leq T)=1-E_{P}\left[1_{\{S \leq T\}}\right] \\
& =E_{P}[B(S)]-E_{P}[B(T)]=E_{P}[B(S)-B(T)] \\
& =E_{P}\left[(B(S)-B(T)) 1_{\{S \leq T\}}\right]=E_{P}\left[(B(S)-B(T))^{+}\right],
\end{aligned}
$$

where $a^{+}=a \vee 0$.

This expression is an extension of the Barlow and Proschan reliability importance, by Bueno and Menezes [5] where the importance of the component $\mathrm{S}$ for the system is $E_{P}\left[(B(S)-B(T))^{+}\right]$.

Now, we ask how we can use Corollary 2.4 to calculate the Barlow and Proscha relibility importance of component $S$ for the system reliability. In a series representation we have:

$$
\begin{aligned}
& I_{Q}(S)=Q(S=S \wedge T)=Q(S \leq S \wedge T) \\
& =Q(S \leq T)=E_{Q}\left[1_{\{S \leq T\}}\right]=E_{Q}\left[N_{B}(T)\right]=E_{P}\left[L(\infty) N_{B}(T)\right] \\
& =E_{P}\left[N_{B}(T)\right]=E_{P}\left[1_{\{S \leq T\}}\right]=1-I_{P}(S) .
\end{aligned}
$$

In the above we use that

$$
\left|E_{P}\left[L(\infty) N_{B}(T)-N_{B}(T)\right]\right| \leq E_{P}\left[\left|(L(\infty)-1) N_{B}(T)\right|\right]
$$

and

$$
E_{P}\left[\left|(L(\infty)-1) N_{B}(T)\right|\right] \leq E_{P}[L(\infty)-1]=0 .
$$

We expected such a relation since that the reliability of the dual system is $\Phi^{D}(X(t))=1-\Phi(1-X(t))$, and the measure $Q$ is relative to the random vector $1-X(t)$. To clarify this argument, suppose that we can define the reverse times, $S^{*}$ and $T^{*}$ such that the events $\left\{S^{* \prime} \leq t\right\}$ and $\left\{T^{* \prime} \leq t\right\}$ are equivalent to $\{S>t\}$ and $\{T>t\}$ respectively, then

$$
\begin{aligned}
& Q\left(S^{* \prime} \leq S^{* \prime} \wedge T^{* \prime}\right) \\
& =Q\left(S^{* \prime} \leq T^{* \prime}\right)=Q(S>T)=1-Q(S \leq T)=I_{P}(S) .
\end{aligned}
$$

\section{A General Parallel System}

The structural relationship between the lifetime $T$, of a parallel system and its components lifetime $T_{1}, T_{2}, \cdots, T_{n}$ is given by

$$
T=\max _{1 \leq i \leq n} T_{i}=\coprod_{1 \leq i \leq n} T_{i} .
$$

We intend to define a compensator transform to characterize the parallel system as a series system. As in Section 2, the idea is to combine compensator transformations on $A_{i}(t)$ the compensator process of the lifetime counting failure process $1_{\left\{T_{i} \leq t\right\}}$ of component $i, 1 \leq i \leq n$. For $1 \leq l \leq n$ define the compensator transform $A_{l}^{*}(t)=\int \alpha_{l}(s) \mathrm{d} A_{l}(s)$, where

$$
\alpha_{l}(s)=\frac{\sum_{k=1}^{n}(-1)^{(k-1)} \sum_{1 \leq i_{1} \leq \cdots \leq i_{n-k} \leq n ; i_{j} \neq l} \mathrm{e}^{\sum_{J=1}^{N-K} A_{i j}(s)}}{D(s)}
$$

and

$$
D(s)=\sum_{k=1}^{n}(-1)^{(k-1)} \sum_{1 \leq i_{1} \leq \cdots \leq i_{n-k} \leq n ; i_{j} \neq 1} \mathrm{e}^{\sum_{J=1}^{N-K} A_{j j}(s)} .
$$

Under the above hypothesis and notation we have

$$
\sum_{l=1}^{n} A_{l}^{*}=\sum_{l=1}^{n} A_{l}-\ln D(t) .
$$

The main results, which follows from an adaptation of Girsanov Theorem, is

\section{Theorem 3.1}

Under the above hypothesis the following process

$$
\begin{aligned}
& L(t)=\prod_{l=1}^{n}\left(\alpha_{l}\left(T_{l}\right)\right)^{1_{\{}\{\leq t\}} \mathrm{e}^{\left[\sum_{j=1}^{n} A_{j}(t)-\sum_{j=1}^{n} A_{j}^{*}(t)\right]} \\
& =\prod_{l=1}^{n}\left(\alpha_{l} T_{l}\right)^{1_{\{}\left\{T_{l} \leq t\right\}} \sum_{k=1}^{n}(-1)^{k-1} \sum_{1 \leq i_{1} \leq \cdots \leq i_{n} \leq n} \mathrm{e}^{\sum_{J=1}^{n-k} A_{j}(t)}
\end{aligned}
$$

is a nonnegative $\mathfrak{I}_{t}$-martingale with $E[L(\infty)]=1$.

\section{Remarks:}

1) We have

$$
L(\infty)=\prod_{l=1}^{n}\left(\alpha_{l}\left(T_{l}\right)\right) \sum_{k=1}^{n}(-1)^{k-1} \sum_{1 \leq i_{1} \leq \cdots \leq i_{n} \leq n} \mathrm{e}^{\sum_{j=1}^{n-k} A_{j}(T)} .
$$

2) If the components are dependent but identically distributed, we have

$$
\begin{aligned}
D(t) & =\sum_{k=1}^{n}(-1)^{(k-1)} \sum_{1 \leq i_{1} \leq \cdots \leq i_{n-k} \leq n ; i_{j} \neq l} \mathrm{e}^{\sum^{n-k=1} A_{j j}(t)} \\
& =\sum_{k=1}^{n}(-1)^{(k-1)} \sum_{1 \leq i_{1} \leq \cdots \leq i_{n-k} \leq n ; i_{j} \neq l} \mathrm{e}^{[(n-k) A(t)]} \\
& =\sum_{k=1}^{n} \frac{n !}{(n-k) ! k !} \mathrm{e}^{[(n-k) A(t)]} \\
& =\sum_{j=0}^{n-1} \frac{n !}{(n-j) ! j !} \mathrm{e}^{[j A(t)]}=\mathrm{e}^{[n A(t)]}-\left(\mathrm{e}^{[A(t)]}-1\right)^{n},
\end{aligned}
$$

and

$$
\begin{aligned}
& \sum_{k=1}^{n}(-1)^{k-1} \sum_{1 \leq i_{1} \leq \cdots \leq i_{n-k} \leq n ; i_{j} \neq 1} \mathrm{e}^{\left[\sum_{j=1}^{n-k} A_{j j}(s)\right]} \\
& =\sum_{j=0}^{n-1}(-1)^{n-j-1} \frac{(n-1) !}{(n-1-j) ! j !} \mathrm{e}^{[j A(t)]}=\left(\mathrm{e}^{\mathrm{A}(t)}-1\right)^{n-1} .
\end{aligned}
$$


Therefore

$$
\alpha_{l}(t)=\frac{\left(\mathrm{e}^{[A(t)]}-1\right)^{n-1}}{\mathrm{e}^{\left[{ }^{[n A(t)]}-\left(\mathrm{e}^{A(t)}-1\right)^{n}\right.} .}
$$

\section{Remark:}

As in Barlow and Proschan [1], we assume the series parallel decomposition of a coherent system:

$$
\Phi(X(t))=\min _{1 \leq j \leq k} \min _{i \in K_{j}} X_{i}(t),
$$

where $K_{j}, 1 \leq j \leq k$, are minimal cut sets, that is, a minimal set of components whose joint failure causes the system to fail.

We can also define, for each $j, 1 \leq j \leq k$, the minimal parallel cut structure

$$
\Gamma_{j}(X(t))=\max _{i \in K_{j}} X_{i}(t),
$$

and we can write

$$
\Phi(X(t))=\min _{1 \leq j \leq k} \Gamma_{j}(X(t)) .
$$

Therefore, using the compensator series transformation for each $\Gamma_{j}$ we get the series transformation for the system.

\section{Preservations Results}

In many reliability situations, we encounter structures of coherent systems where components share a load, so that a failure of one component results in increased load on each of the remaining components. Furthermore, the components in a coherent system could be subjected to the same set of stress. In such cases, the random variables of interest are not independent but rather associated.

Therefore, it is very interesting to verify whether the association properties of the lifetimes $T_{1}, \cdots, T_{n}$ under $P$ are preserved under $Q$.

We introduce the association definition. For this we give the concept of an upper set in $\mathfrak{R}^{n}$.

A Borel set $U \subset \mathfrak{R}^{n}$ is called an upper set if for any $x, y \in \mathfrak{R}^{n}, x \leq y, x \in U$ implies that $y \in U$. In the univariate case, $U$ is equal to either $(u, \infty)$ or $[u, \infty)$.

\section{Definition 4.1}

The random variables $T_{1}, \cdots, T_{n}$ (or the corresponding random vector $T$ ) are associated, if for all upper sets $U_{1}$ and $U_{2}$ of $\mathfrak{R}^{n}$

$$
P\left(T \in U_{1} \cap U_{2}\right) \geq P\left(T \in U_{1}\right) P\left(T \in U_{2}\right) .
$$

In particular, this definition, formulated by Esary, Proschan and Walkup [6], Esary and Proschan [7], is useful to produce upper and lower bounds for system reliability. The measure $Q$ preserves this property from the measure
$P$.

\section{Theorem 4.2}

If $T$ is associated under $P$, then also under $Q$. Proof

We consider the upper sets in $\mathfrak{R}^{n}, U_{1}$ and $U_{2}$, and the uniformly integrable $\mathfrak{J}_{t}$-martingale,

$$
M^{1}(t)=E_{p}\left[1_{U_{1}}(T) \mid \mathfrak{J}_{t}\right]
$$

and

$$
M^{2}(t)=E_{p}\left[1_{U_{2}}(T) \mid \Im_{t}\right] .
$$

It follows that there exists a unique $\mathfrak{J}_{\text {t }}$-predictable process, such that the covariance process $\left\langle M^{1}, M^{2}\right\rangle(t)$ is increasing, right continuous, with $\left\langle M^{1}, M^{2}\right\rangle(0)=0$ such that

$$
M(t)=M^{1}(t) M^{2}(t)-\left\langle M^{1}, M^{2}\right\rangle(t)
$$

is an $\mathfrak{J}_{t}$-martingale.

Since a martingale has a constant expectation, we have

$$
\begin{aligned}
E_{P}[M(t)] & =E_{P}[M(0)] \\
& =E_{P}\left[M^{1}(0) M^{2}(0)\right] \\
& =E_{P}\left\{E_{P}\left[1_{U_{1}}(T) \mid \mathfrak{I}_{0}\right] E_{P}\left[1_{U_{2}}(T) \mid \mathfrak{I}_{0}\right]\right\} \\
& =P\left(T \in U_{1}\right) P\left(T \in U_{2}\right)
\end{aligned}
$$

and

$$
\begin{aligned}
& E_{P}\left[\left\langle M^{1}, M^{2}\right\rangle(\infty)\right] \\
& =P\left(T \in U_{1} \cap U_{2}\right)-E_{P}\left[\left\langle M^{1}, M^{2}\right\rangle(\infty)\right] \\
& =P\left(T \in U_{1}\right) P\left(T \in U_{2}\right) .
\end{aligned}
$$

It follows that $T$ is associated if, and only if,

$$
\begin{aligned}
& E_{P}\left[\left\langle M^{1}, M^{2}\right\rangle(\infty)\right] \\
& =P\left(T \in U_{1} \cap U_{2}\right)-P\left(T \in U_{1}\right) P\left(T \in U_{2}\right) \geq 0 .
\end{aligned}
$$

Now,

$$
E_{Q}\left[\left\langle M^{1}, M^{2}\right\rangle(\infty)\right]=E_{P}\left[L(\infty)\left\langle M^{1}, M^{2}\right\rangle(\infty)\right] \geq 0
$$

for $L(\infty) \geq 0 \quad P$ a.s.

Classes of non-parametric distributions, such as increasing ( decreasing) failure rate (IFR (DFR)) distributions, new better (worse) than used (NBU (NWU)) distributions and others, have been extensively investigated in Reliability Theory. They can be used to achieve the benefit of a maintenance operation or to derive bounds on system reliability.

Several extensions of these concepts appeared in the literature, e.g. Harris [8], Barlow and Proschan [3], Marshall [9] and others. However, they all have in common that they don't order the lifetime vectors in the sense of 
stochastic order as the univariate concept does. Arjas [10], considered to observe the components, continuously in time, based on a family of sub $\sigma$-algebras $\left(\mathfrak{J}_{t}\right)_{t \geq 0}$. Arjas, introduced the notion of increasing failure rate distribution (new better than used distribution) relative to $\left(\mathfrak{J}_{t}\right)_{t \geq 0} \operatorname{IFR} \mid \mathfrak{I}_{t},\left(\mathrm{NBU} \mid \mathfrak{I}_{t}\right)$ generalizing the conventional definition of IFR (NBU) and extending these classes into a multivariate form, denoted by $\operatorname{MIFR} \mid \mathfrak{I}_{t},\left(\mathrm{MNBU} \mid \mathfrak{I}_{t}\right)$.

In order to introduce the concepts of Arjas [10], we define the residual lifetime of $T_{i}$ at time $t, 1 \leq i \leq n$ as

$$
\theta_{t} T_{i}=\left(T_{i}-t\right)^{+}=\max \left\{T_{i}-t, 0\right\} .
$$

Let $\theta_{t} T=\left(\theta_{t} T_{1}, \cdots, \theta_{t} T_{n}\right)$.

\section{Definition 4.3}

(Arjas, [10]) We say that $T$ is multivariate increasing failure rate relative to $\left(\mathfrak{I}_{t}\right)_{t \geq 0}$, denoted by $\operatorname{MIFR} \mid \mathfrak{I}_{t}$, if for all $0 \leq s \leq t$ and all open upper sets $U \subset \mathfrak{R}^{n}$,

$$
P\left(\theta_{t} T \in U \mid \mathfrak{I}_{t}\right) \leq P\left(\theta_{s} T \in U \mid \mathfrak{I}_{t}\right) .
$$

Remark: Arjas [1] proved that the class of upper sets in the above definition can be restrict to the class $U_{Q}$, of finite unions of open upper sets with corner point $q=\left(q_{1}, \cdots, q_{n}\right)$, where $q_{i}, 1 \leq i \leq n$ are positive rational numbers. An open upper set with corner point $q=\left(q_{1}, \cdots, q_{n}\right)$ is defined by $U_{q}=\prod_{i=1}^{n}\left(q_{i}, \infty\right)$.

We want to prove that the MIFR $\mid \mathfrak{J}_{t}$ class under $P$ is preserved under $Q$.

\section{Theorem 4.4}

If $T$ is multivariate IFR relative to $\left(\mathfrak{J}_{t}\right)_{t \geq 0}$, under $P$, then also under $Q$.

Proof First, we show that $T_{i}$ is $\operatorname{IFR} \mid \mathfrak{J}_{t}$, under $Q$. By hypothesis, we have

$$
\int_{A} P\left(\left(T_{i}-t\right)^{+}>s \mid \mathfrak{J}_{t}\right) \mathrm{d} P=E_{P}\left[1_{A} 1_{\left\{T_{i}>t+s\right\}}\right] \downarrow t
$$

for all $A \in \mathfrak{I}_{t}$.

Now

$$
\begin{aligned}
& \int_{A} Q\left(\left(T_{i}-t\right)^{+}>s \mid \mathfrak{J}_{t}\right) \mathrm{d} Q=E_{Q}\left[1_{A} 1_{\left\{T_{i}>t+s\right\}}\right] \\
& =E_{P}\left[L(\infty) 1_{A} 1_{\left\{T_{i}>t+s\right\}}\right] .
\end{aligned}
$$

However $L(\infty)$ is a measurable random variable and can be written as a suitable approximating step function $\sum_{i=1}^{n} \alpha_{i, n} 1_{A_{i, n}}$, where $\alpha_{i, n}$ are constants and $A_{i, n}$ are measurable.

As $E_{P}[L(\infty)]=1$, using the dominated convergence theorem we have

$$
\int_{A} Q\left(\left(T_{i}-t\right)^{+}>s \mid \mathfrak{I}_{t}\right) \mathrm{d} Q=\lim _{n \uparrow \infty} \sum_{i=1}^{n} \alpha_{i, n} E_{P}\left[1_{A_{i, n}} 1_{A} 1_{\left\{T_{i}>t+s\right\}}\right]
$$

which is decreasing by hypothesis.

Herefore $T_{i}$ is IFR $\mid \mathfrak{J}_{t}$ under $Q$. In order to prove that the vector $T$ is increasing failure rate relative to $\left(\mathfrak{J}_{t}\right)_{t \geq 0}$ we use the relation

$$
\left\{\left(T_{1}-t\right)^{+}>s, \cdots,\left(T_{n}-t\right)^{+}>s\right\}=\bigcap_{1 \leq i \leq n}\left\{\left(T_{n}-t\right)^{+}>s\right\}
$$

and the equivalent definition of MIFR $\mid \mathfrak{I}_{t}$ in terms of the open upper sets with corner point as in the above remark.

As $T$ is $\operatorname{MIFR} \mid \mathfrak{J}_{t}$,

$$
\int_{A} P\left(\bigcap_{1 \leq i \leq n}\left(T_{n}-t\right)^{+}>s \mid \mathfrak{I}_{t}\right) \mathrm{d} P=E_{P}\left[1_{A} \prod_{i=1}^{n} 1_{\left\{T_{i}>t+s\right\}}\right] \downarrow t .
$$

The proof follows as above.

\section{Conclusions}

Relay system are very concerned in reliability theory, however, such a modeling is complicated under stochastically dependence conditions. The purpose of this paper is to provide a way to work with this situation using a point process martingale approach.

Some preservation important results in association and non parametric distributions classes useful in reliability theory are proved and an application in component importance is analised.

\section{Acknowledgments}

This work was partially supported by FAPESP, Proc. No. 2010/52227-0.

\section{REFERENCES}

[1] R. Barlow and F. Proschan, "Statistical Theory of Reliability and Life Testing: Probability Models,” Mc Ardle Press, Inc. Silver Spring, 1981.

[2] P. Bremaud, "Point Processes and Queues: Martingales Dynamics,” Springer Verlag, New York, 1981. doi:10.1007/978-1-4684-9477-8

[3] E. Arjas and A.Yashin, "A Note on Random Intensities and Conditional Survival Functions,” Journal of Applied Probability, Vol. 25, No. 3, 1988, pp. 630-635. doi:10.2307/3213991

[4] V. C. Bueno and I. M. Carmo, "Active Redundancy Allocation for a $k$-Out-Of- $n$ :F System of Dependent Components," European Journal of Operational Research, Vol. 176, No. 2, 2007, pp. 1041-1051. doi:10.1016/j.ejor.2005.09.012

[5] V. C. Bueno and J. E. Menezes, "Patterns Reliability Importance under Dependence Condition and Different Information Levels," European Journal of Operational Research, Vol. 177, No. 1, 2007, pp.354-364. doi:10.1016/j.ejor.2005.10.042

[6] J. D. Esary, F. Proschan and D. W. Walkup, “Association of Random Variables with Applications,” The Annals of 
Mathematical Statistics, Vol. 38, No. 5, 1967, pp.14661474. doi:10.1214/aoms/1177698701

[7] J. D. Esary and F. Proschan, "A Reliability Bound for System of Maintained, Interdependent Components," Journal of American Statistical Association, Vol. 65, No. 329, 1970, pp.713-717.

[8] R. Harris, "A Multivariate Definition for Increasing Hazard Rate Distribution Functions," The Annals of Mathematical Statistics, Vol. 41, No. 2, 1970, pp. 713-717. doi:10.1214/aoms/1177697121
[9] A. W. Marshall, "Multivariate Distributions with Monotone Hazard Rate,” In: R. E. Barlow, J. B. Fussel and N. D. Singpurwalla, Eds., Reliability and Fault Tree Analysis, Society for Industrial and Applied Mathematics, Philadelphia, 1975, pp. 259-284.

[10] E. Arjas “A Stochastic Process Approach to Multivariate Reliability System: Notions Based on Conditional Stochastic Order," Mathematics of Operations Research, Vol. 6, No. 2, 1981, pp. 263-276. doi:10.1287/moor.6.2.263 